\title{
A Dynamic Network Reconstruction Approach using ACO
}

\author{
Anamika $^{1}$, Neeru Singla ${ }^{2}$, Neha Sharma ${ }^{3}$ \\ ${ }^{1}$ M.Tech Student, Department of Electronics and Communication Engineering \\ ${ }^{2,3}$ Assistant Professor, Rayat Institute of Engineering and Information Technology, Punjab Technical \\ University, Punjab (India)
}

\begin{abstract}
Energy efficiency and reliability are always the main concern in wireless sensor networks. In a sensor network the nodes are present with limited energy and with each transmission they loss some energy. It is required to minimize the rerouting to save the energy loss. We proposed an algorithm to get the efficiency as well as the reliability. An algorithm for energy efficient maximally covered sensor network is presented. In this work the Path Selection Routing along with the concept of Ant Optimization is combined. The initial route will be identified by the Path Selection algorithm and in case of any broken link or intrusion in the path it will look for the alternate path using Ant Optimization. The performance is evaluated with parameters. In conclusion, a comparison between proposed work and literature proves the efficiency of our work.
\end{abstract}

Keywords: ACO, Energy Optimization, Path Selection Algorithm, Wireless Sensor Network (WSN)

\section{Introduction}

The advances in Wireless communication and electronics brought new research in Wireless Sensor Networks. Wireless Sensor Networks (WSN) has gained world-wide attention in recent years. It is a sensing technology where autonomous devices called sensor nodes are deployed in a remote area to observe phenomena, collect data and process it and then transmit information to users via radio frequency (RF) channel. The concept of wireless sensor networks is based on a simple equation: Sensing + CPU + Radio $=$ Thousands of potential applications. With the advances in processor, memory, and wireless communication, Wireless Sensor Networks (WSNs) has been emerged as a promising technology to help human for performing many tasks such as environment and habitat monitoring, health-care application, traffic control, or wild ecological survey. Although WSN is used in many applications, it has many restrictions such as limited energy supply, limited computation and limited communication abilities. So various routing protocols have been designed and developed for WSNs because the routing in WSNs is distinguished from other networks. First, due to the relatively large number of sensor nodes, it is impossible to build a global addressing scheme for the deployment of a large number of sensor nodes. Thus, traditional Internetworking Protocol (IP) based protocols may not be applied to WSNs. Second, sensor nodes are tightly constrained in terms of energy, processing, and storage capacities. So they require careful resource management. Third, as node failure occurs frequently in WSNs which results in unpredictable and frequent topological changes. So the routing protocol must adapt to frequent changes of the WSNs topology. Sensor nodes in WSNs are small and compact in size and are capable of sensing the surroundings, gathering data and then processing it for communicating with other connected nodes in the network. Sensor networks are composed of multiple interconnected and distributed sensors that collect information on areas or objects of interest. Sensor nodes make up each sensor network and consist of three major components - object, event and parameter sensing, data processing and classification and data communications.

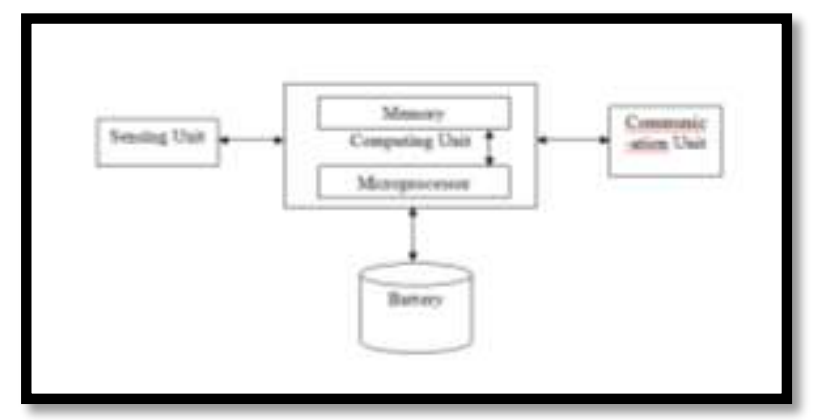

Figure1. Architecture of Wireless Sensor Node

The basic block diagram of a wireless sensor node is presented in Fig. 1. It is made up four basic components: a sensing unit, a processing unit, communication link and battery. 


\subsection{Overview of ACO Based Routing Algorithms in WSN.}

Sanjoy Das et al have given an on-line ACO algorithm using AntNet techniques for MSDC [15] which has been formalized to be a typically Minimum Steiner Tree problems. They also have proposed an improved algorithm by adding another type of ants, random ants, analogous to a newspaper deliverer, whose main task is to disperse information gathered at the nodes among other neighboring nodes. Practically, simulation results also show that their algorithms are significantly better than address-centric routing. In these proposed algorithms the forward ants normally spend a long time.

There is a bug of dead lock in their algorithms. In their improved algorithm, a large number of random ants are needed. In [16] the authors propose a new idea of keeping the information by all sensor nodes of their own. By this even in the absence of global processing the nodes still can work on their own information. This still has the drawback of broadcasting in the initialization phase, which consumes lots of energy at the beginning of the network deployment. Zhang et al. [17] proposed three ant-routing algorithms for sensor networks. The SC algorithm is energy efficient but suffers from a low success rate. The FF algorithm has shorter time delays; however, the algorithm creates a significant amount of traffic. Despite high success rate shown by the FP algorithm but is not energy efficient. An Adaptive ant-based Dynamic Routing (ADR) algorithm using a novel variation of reinforcement learning was proposed by Lu et al. [18]. The authors used a delay parameter in the queues to estimate the reinforcement learning factor. Karaboga [21] proposed a novel approach for WSN routing operations. Through this approach the network life time is maximized, for discovering the shortest paths from the source nodes to the base node using an evolutionary optimization technique. The research has also been implemented on the PIC ${ }^{\circledR}$ series of microcontrollers, specifically the PIC12F683. Aghaei et al [19] proposes two adaptive routing algorithms based on ant colony algorithm, the Adaptive Routing (AR) algorithm and the Improved Adaptive Routing (IAR) algorithm. To check the suitability of the ADR algorithm in the case of sensor networks, they modified the ADR algorithm (removing the queue parameters) and used their reinforcement learning concept and named it the AR algorithm. The AR algorithm did not result in optimum solutions. In IAR algorithm by adding a coefficient, the cost between the neighbor node and the destination node, they further improve the AR algorithm. Wen et al [20] proposed a dynamic adaptive ant algorithm (E\&D ANTS) is based on Energy and Delay metrics for routing operations. Their main goal is to maximize the network lifetime while minimizing propagation delay by using a novel variation of reinforcement learning (RL). E\&D ANTS results was evaluated with AntNet and AntChain schemes.

\section{Literature Review}

Many researchers in the past have tried to improve WSNs using various algorithms and techniques.

\subsection{Path Selection Algorithm [5] -}

The energy should be under consideration when a routing protocol is designed for the wireless sensor networks. The reason is that energy retention of the network is directly related to the network efficiency. Therefore, minimizing the energy consumption is an important factor for the protocol design. In PSA, both minimization of the energy required for transmission, and available energy in the nodes are considered when deciding a "right path". A "right path" means that among many possible paths, it is a path consisting of the nodes that have enough energy for transmission and it has the highest selectivity. The available energy Ea,pi for a particular path $p i$ is defined as the sum of the available energy of all nodes on that path. Whenever a particular source sends a packet to the same destination, a path is required from the source to the destination. Therefore, the concept of round can be brought in. A "right path" among many paths is found by considering all the factors such as node energy and the number of times this path has been selected. This is a round 1 and it continues to go on whenever the source needs a path to the same destination for transmission. MTE protocol: min $(E c, p 1, \ldots$ $, E c, p n) E c, p i$ denotes the energy consumed for transmission in a certain path $p i$. Available energy: $E a, p 1, E a, p 2$, $E a, p 3, \ldots E a, p n$. There are two requirements on how a particular path $p i$ is selected: $E a, p i>E c, p i$ (1)

$S p i=P(p i) / 1-P(p i) \times(k \bmod 1 / P(p i)) . E a, p i / E m, p i(2)$

In the first requirement, it shows that the available energy in a certain path should be larger than the energy consumed for transmission. This is an obvious condition that must be satisfied; otherwise the transmission would be aborted on the way before the destination. In the second requirement, Spi stands for selectivity of the path pi being selected as a right path. Among all the values of the selectivity, the path that has the largest value will be selected as a right path. The maximum value of the selectivity is $1 . P(p i)$ is the desired probability for the path pi. Normally, it is determined depending on the number of paths, $\mathrm{n}$ found by the route discovery procedure. In this case, the desired probability is set to $1 / \mathrm{n}$ for each path, so that all the discovered paths can be used equally, thereby maximizing the network lifetime in terms of energy. On the other hand, it is possible to give priority to some path by increasing the desired probability. For example, if the traffic type is delay-sensitive (e.g., urgent event), priority is given to the path with shortest delay, so that this path can be 
chosen first. Also, if a certain path retains the available energy close to the consumption energy, the desired probability is lowered enough to prevent this particular path from being selected, because it would cause an energy drain of the path once used. Besides the desired probability, the other factor that affects the path selectivity $\mathrm{S}$ is the round $\mathrm{k}$ in the first term of the equation above. If a source has discovered $\mathrm{n}$ paths to a given destination, one cycle is formed with $\mathrm{n}$ rounds between these two nodes, and the desired probability is also determined at this point. Normally, the value of the desired probability is changed on a cycle basis. Each cycle starts from round 0 and ends with round $n-1$. Whenever one of $n$ paths is used to send a packet to the destination, the round value increases by one until the last round $n-1$. Once a certain path is chosen, the selectivity of that path is set to 0 so that it cannot be used again during this cycle. For any other path, the selectivity continues to increase according to the increment of the round value, as long as it is not chosen. Finally, the first term of the selectivity equation approaches to one so that it will be chosen during this cycle. The reason why a certain path even with the higher available energy is used only once at each cycle is because the environment of wireless sensor networks is changing fast in time due to the wireless links, mobility, or node energy consumption for local processing and so on. Therefore, if the source has another data to send to the same destination after one cycle ends, it executes the route discovery procedure again to find new paths and start a new cycle by reflecting the updated network environment. In the second term of Eq. (2), Em,pi represents the maximum energy for the path pi. Therefore, if nodes have low available energy, then the value of this fraction will be small (i.e. close to 0 ), because the value of Ea,pi is small and the value of Em,pi is large. This means if there is not enough available energy, then such a path will not be chosen due to the small value of the selectivity. In contrast, if nodes have full energy, then the value of this fraction will be very large (i.e. close to 1), because the value of Ea,pi approaches to the maximum energy. As a result, such a path is likely to be chosen as a right path unless there is any larger value than this.

\subsection{Ant Colony Optimization [2] -}

Research shows that ants have the ability to select the shortest path among few possible paths connecting their nest to a food site. The pheromone, a volatile chemical substance laid on the ground by the ants while walking and affecting in turn their moving decisions according to its local intensity, is the mediator of this behavior.

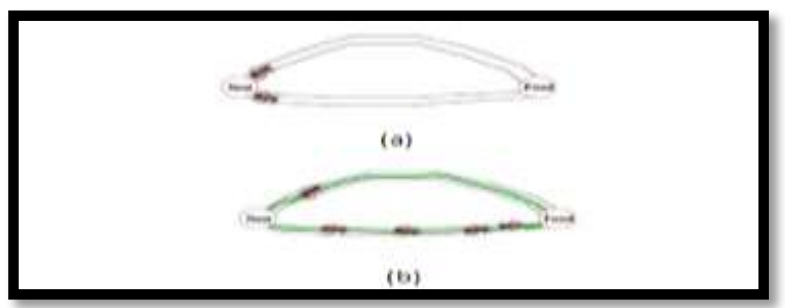

Figure2. Principle of Ant Colony Optimization

As shown in Fig. 2(a), at the beginning, no pheromone is laid on the branches and the ants do not have any bit of information about the branches length. However, since one branch is shorter than the other, the shorter branch receives pheromone at a higher rate than the longer one. As ants can smell pheromone, and their probabilistic decisions are based in favor of paths marked with higher amount of pheromone. Eventually, the shorter path will be selected by almost all ants of the colony (as shown in Fig. 2(b)).Ant colony optimization metaheuristic, a novel population-based approach was recently proposed in 1992 by Marco Dorigo et al. to solve several discrete optimization problems. The ACO mimics the way real ants find the shortest route between a food source and their nest. The ants communicate with one another by means of pheromone trails and exchange information about which path should be followed. The more the number of ants traces a given path, the more attractive this path (trail) becomes and is followed by other ants by depositing their own pheromone.

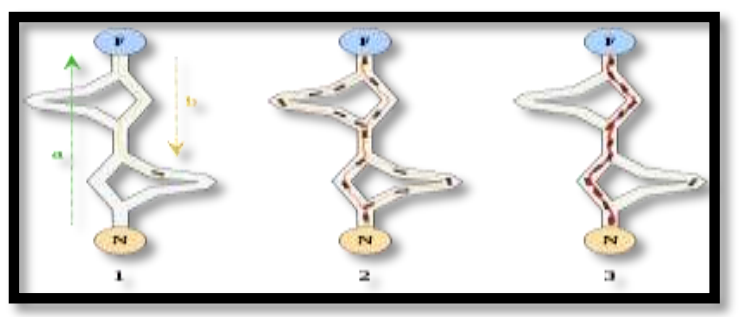

Figure 3.Ant Colony system 
This auto catalytic and collective behavior results in the establishment of the shortest route as shown in Fig. 3. Most ant colony optimization algorithms use this algorithmic diagram demonstrated below:Initiation of the parameters which determines the pheromone trail While (until result conditions supplied) do Generate Solutions Apply Local Search Update Pheromone Trail End

Above complex global behaviors are the result of self-organizing dynamics driven by local interactions and communications among a number of relatively simple individuals. The simultaneous presence of these and other fascinating and unique characteristics have made ant societies an attractive and inspiring model for building new algorithms and new multi-agent systems.

The ant colony optimization algorithm combines such characteristics as quick problem-solving, global optimization as well as the high degree of self-organization. And it is very similar to the requirements of lowpower, self-organization and quickly routing in the wireless sensor networks. This caused us to explore the overall energy balance of the wireless sensor network routing protocol based on ant colony algorithm.

\section{Proposed Approach}

In the present work we have improved the path selection algorithm by using the concept of Ant Colony Optimization.

1. Define N Number of Sensor Nodes in the WSN with specific parameters in terms of energy, transmission rate etc.2. Each Node Ni start Moving in Direction of Specific Direction

3. Find M Neighbor Nodes of Nodes Ni and maintains the respective Information

$$
\text { For }(\mathrm{j}=1 \text { to } \mathrm{M}) \quad\{\text { Maintain Formation }(\mathrm{Ni}, \mathrm{Nj})\}
$$

4. if Data Loss(Ni)> Threshold and Time Delay > Threshold1

/*If Bad Node or Congested Node Occur on Node $\mathrm{i} * /\{$ For $\mathrm{i}=1$ to Mi \{Collect Information (Ni, Neighbor $(\mathrm{Ni}))\}\}$

5. Implement Forward ANT to find the alternate path in each Direction of Neighbor (N (i)).

6. Set the Pheromone on Each Hop and Identify the Possible Path

7. Implement Backward ANT to inform Neighbor Nodes about Backup Path

8. Trace the Pheromones and Communication of New Path

9. Perform the Normal Communication $\}$

The first step is to setup the network with specific parameters.

\subsection{Parameters Included}

4.1.1 Number of Packets: This property represents the number of successful packet delivery for a specific communication.

4.1.2 Number of Packets lost: This parameter will analyze the packet loss over transmission. Due to congestion or any block node there are chances of data loss over the network. It is the decision parameter that will perform the analysis that the next node is a valid node or not.

4.1.3. Packet Delivery Ratio: This parameter defines the ratio of packets transmitted and the packets successfully arrived at the destination. We have analyzed packet delivery ratio on 4 intermediate nodes to identify the problem area over the network.

4.1.4. Time Delay: It defines the delay in the communication. The delay occurs because of congestion over the network.

4.1.5. Energy: As each node in the network is a sensor node, each node is defined with specific energy we have defined 5 Joules to each node. With each communication over the network some energy is lost. If the energy is less than minimum required energy or 0 , the node will be dead itself.

4.1.6. Turn-around Time: It is the actual time taken to perform the communication over the network.

4.2. Description of the Ant Concept

4.2.1 At regular interval any node, $\mathrm{s}$ (Source) is selected to send data to some destination node, $\mathrm{d}$.

4.2.2. Each forward ant selects the next hop node using the routing table information. The next node selected depends on some random way. If all nodes are already visited a uniform selection will be performed. 


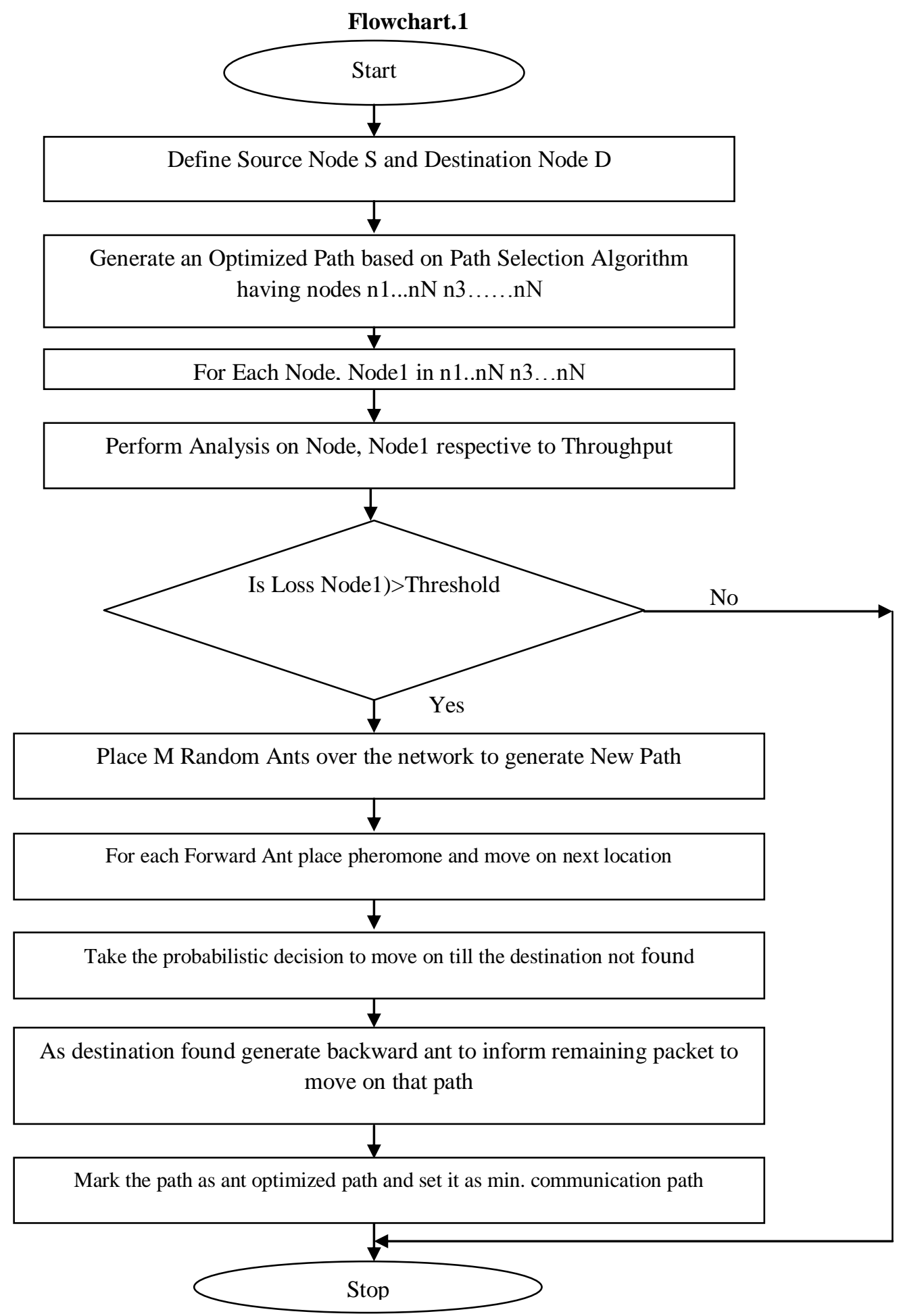

4.2.3. If the selected node is attacked or damaged or node it is not currently available, the forward ant waits to turn in the low priority node from the queue.

4.2.4 It will identify any of the next non-visited node and pay some delay on it.

4.2.5. If some cycle is detected the ant is forced to turn on the visited node.

4.2.6. When the ant reaches the destination node a backward ant is generated to transfer all its memory.

4.2.7. Backward ant uses same path generated by forward ant.

By default route is chosen on the basis of Path selection formula i.e. it will choose the lowest energy path. In case there is problem in the selection of the path then we apply the ACO, the purpose of which is to continue sending data using the previous path. Hence, we achieved efficiency in terms of energy by applying path selection whereas Ant Colony Optimization Algorithm gives the required reliability. 


\section{Results and Discussions}

The basic parameters of the proposed work are presented respective to the simulation environment. The system is implemented on Ubuntu Environment with NS2 simulator and XGraph is used as the tool for graph analysis. The initial energy of each node is 5 Joules.

Table1. Parameters of WSN

\begin{tabular}{|c|c|}
\hline Parameter & Value \\
\hline Number of Nodes & 50 \\
\hline Topography Dimension & $800 \mathrm{~m} \mathrm{x} 800 \mathrm{~m}$ \\
\hline Traffic Type & CBR \\
\hline Radio Propagation Model & Two-Ray Ground Model \\
\hline MAC Type & 802.11 Mac Layer \\
\hline Packet Size & 512 bytes \\
\hline Mobility Model & Random Way Point \\
\hline Antenna Type & Omni directional \\
\hline Protocol & AODV \\
\hline
\end{tabular}

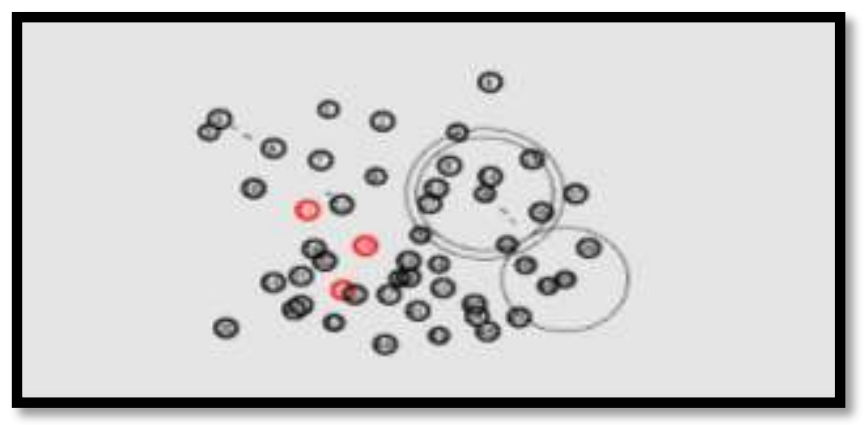

Figure4. Communication from node 0 to 49

The above Fig. 4 shows the basic scenario that is being used in this presented application. The scenario is that of 50 nodes distributed randomly in a work area of $800 \mathrm{~m} \mathrm{x} 800 \mathrm{~m}$. The position of the mobile nodes is defined in terms of $\mathrm{X}$ and $\mathrm{Y}$ coordinate values and it is written in the movement scenario file.

The large circles show the coverage area of the Wireless Sensor Networks. The packets are being transmitted from node 0 to node 49 , the small dashes show the packets being transmitted. Here the path is selected by path selection algorithm i.e. minimum energy path is selected where there is some data loss.

The nodes 11, 29 and 32 are detected as bad nodes (shown in red), i.e. broken link is detected. These bad nodes do not allow to, pass data to the next nodes. A node with less forwarding ratio is presented as a bad node. After the bad nodes were detected, packets changed their path to reach the destination based on ACO without re-routing; therefore energy of the nodes is saved. Packets are also not lost with ACO and therefore energy dissipated is less.

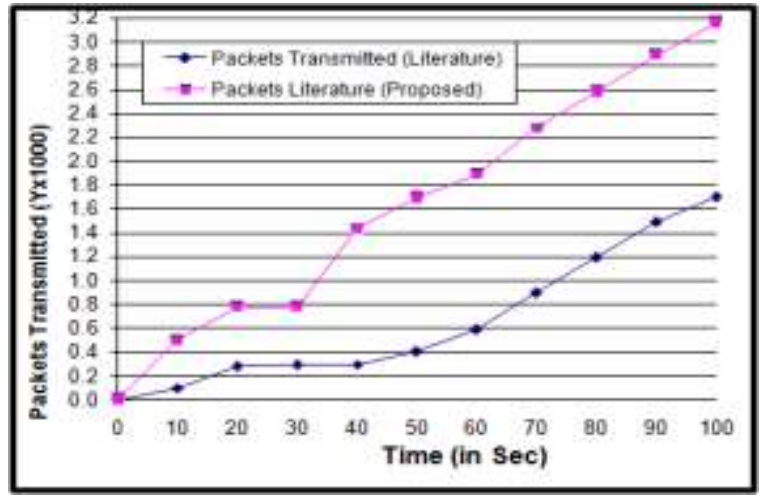

Figure5. Packets Transmitted 
The above Fig. 5 shows the comparative analysis of packets transmitted over the network. After implementing the proposed approach, the number of packets transmitted over the network is increased.

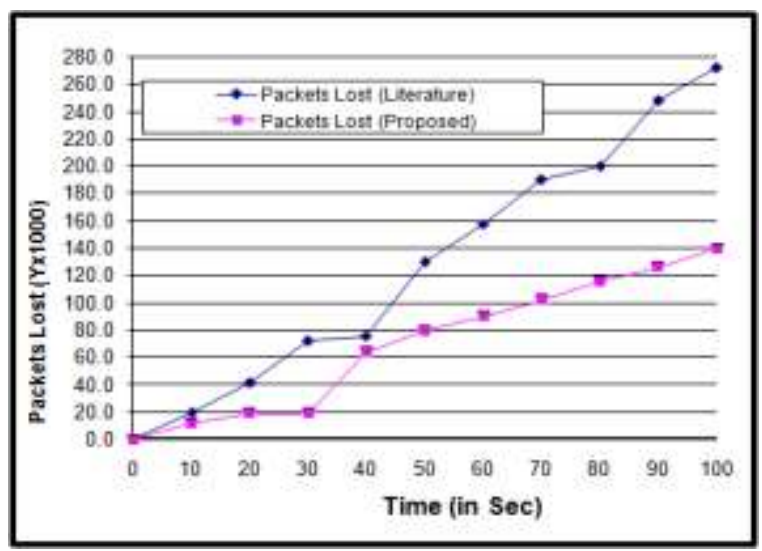

Figure6. Packets Lost

The above Fig. 6 shows the comparative analysis of packet lost over the network. After implementing the proposed approach the packet lost over the network is decreased.

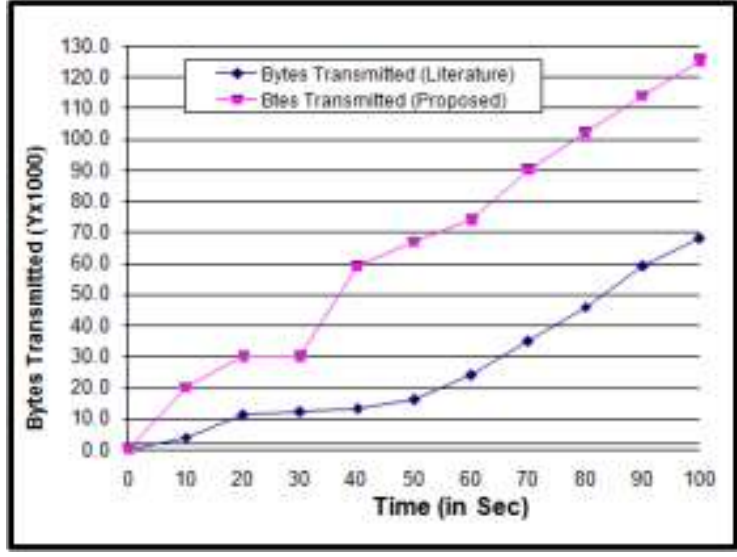

Figure7. Bytes Transmitted

The above Fig. 7 shows the comparative analysis of bytes transmitted over the network. After implementing the proposed approach the bytes transmitted over the network is increased.

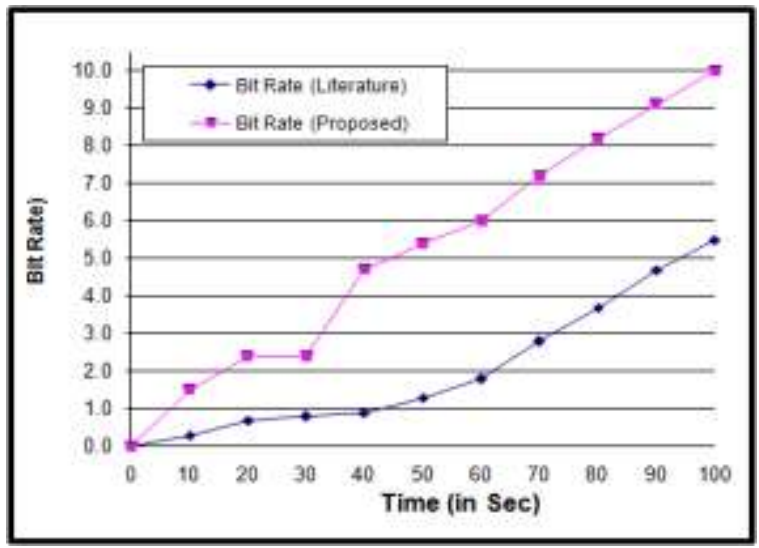

Figure 8.Bit Rate

The above Fig. 8 shows the comparative analysis of bit rate over the network. After implementing the proposed approach the bit rate over the network is increased. 


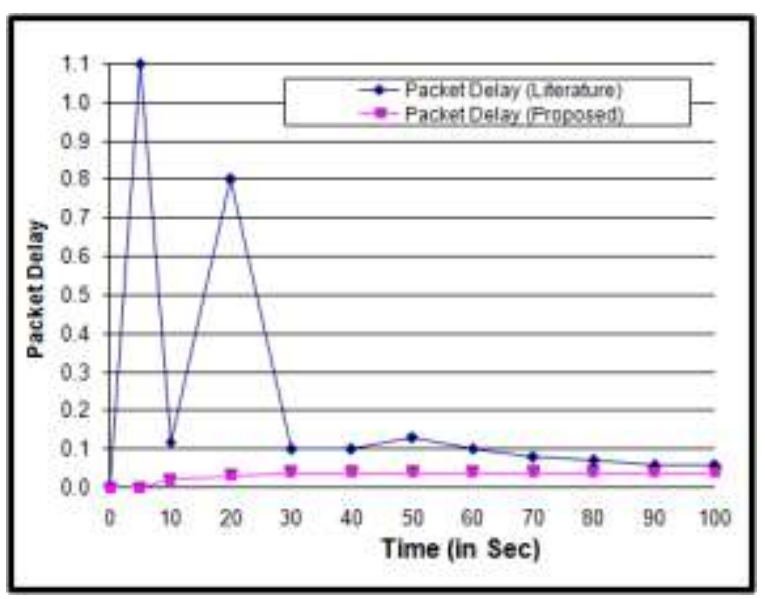

Figure9. Packet Delay

The above Fig. 9 shows the comparative analysis of packet-delay over the network. After implementing the proposed approach the packet-delay over the network is decreased.

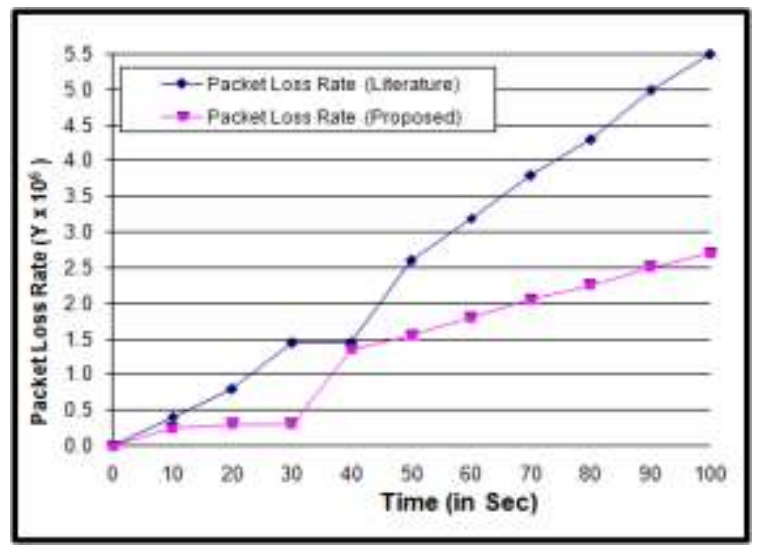

Figure10. Packet Loss Rate

The above Fig. 10 shows the comparative analysis of packet-loss rate over the network. After implementing the proposed approach the packet loss rate over the network is decreased.

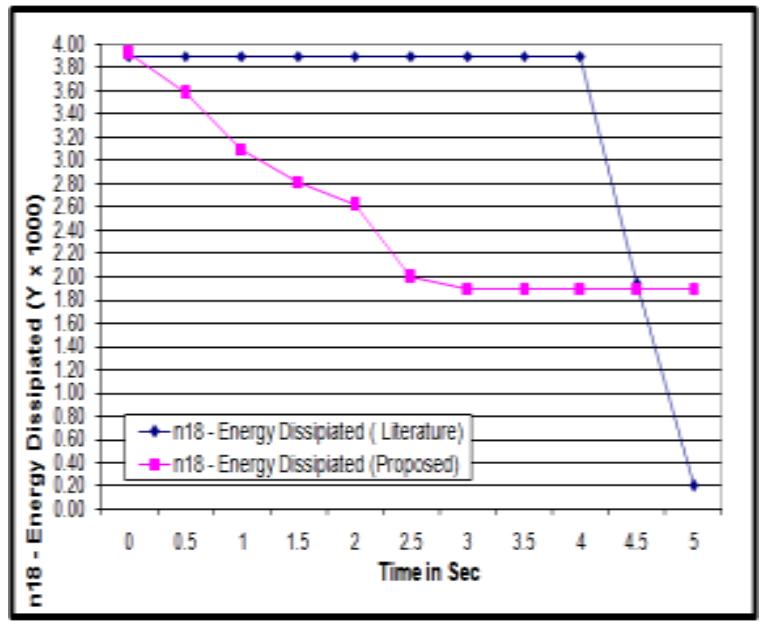

Figure11. Energy Dissipated 
The above Fig. 11 shows the comparative analysis of energy dissipated from node 18 for the existing and the proposed work. With each communication some energy is dissipated. As we can see in this present work the energy dissipated is low as compared to existing approach and also decreases with time.

\section{Conclusion}

By simulation, we have found that the energy in our protocol is dissipated less than the other energyaware routing protocols. By default the route is chosen on the basis of Path Selection formula i.e. lowest energy path is chosen. In case there is problem in the selection of the path (in case of any fault node) then ACO Algorithm is applied, the purpose of which is to continue sending data without re-routing. This ensures the reliability of the network communication i.e. data exchange will not stop even in case of failure of any node. Hence we achieved efficiency in terms of energy by applying path selection whereas Ant Colony Optimization Algorithm gives the required reliability. Implementation is done in NS 2.34.

\section{Journal Papers:}

\section{References}

[1] Young-Min Kim, Eun-Jung Lee And Hong-Shik Park, “Aco Based Energy Saving Routing For Energy-Efficient Networks”, Ieee Communication Letters Vol.15, No.7, July 2011.

[2] Xie Hui, Zhang Zhi-Gang, Nie Feng, "A Novel Routing Protocol In Wireless Sensor Networks Based On Ant Colony Optimization”, International Journal Of Intelligent Information Technology Application, 2010, 3(1):1-5.

[3] Ahmed A. A. Zakzouk, "An Ant Colony Optimization Approach For Solving Shortest Path Problem With Fuzzy Constraints".

[4] Afshin Ghanizadeh, "A Fuzzy-Particle Swarm Optimization Based Algorithm For Solving Shortest Path Problem”, 978-1-42446349-7/10@2010 Ieee.

[5] Inwhee Joe, "A Path Selection Algorithm With Energy Efficiency For Wireless Sensor Networks", Fifth International Conference On Software Engineering Research, Management And Applications.

[6] Michael Rinehart, "The Value Of Sequential Information In Shortest Path Optimization", 2010 American Control Conference Marriott Waterfront, Baltimore 978-1-4244-7427-1/10@2010 Aacc.

[7] Yanfang Deng, "Dynamic Shortest Path In Stochastic Traffic Networks Based On Fluid Neural Network And Particle Swarm Optimization”, 2010 Sixth International Conference On Natural Computation (Icnc 2010) 978-1-4244-5961-2/10@2010 Ieee.

[8] Maumita Bandyopadhyay, "Zone Based Ant Colony Routing In Mobile Ad-Hoc Network", 978-1-4244-5489-1/10@ 2010 Ieee.

[9] Marina Yusoff, “A Discrete Particle Swarm Optimization With Random Selection Solution For The Shortest Path Problem", 978-14244-7896-5/10@2010 Ieee.

[10] Zhang Huidang, "The Particle Swarm Optimization With Decaying Icmic For Shortest Path Computation In Computer Networks", 2010 International Conference On Computational Intelligence And Security 978-0-7695-4297-3/10@ 2010 Ieee.

[11] Je'Ro`Me Berclaz, "Multiple Object Tracking Using K-Shortest Paths Optimization”, Ieee Transactions On Pattern Analysis And Machine Intelligence 0162-8828/11@ 2011 Ieee.

[12] Kavitha Bhaskaran, "Dynamic Anycast Routing And Wavelength Assignment In Wdm Networks Using Ant Colony Optimization (Aco)", Ieee Icc 2011 978-1-61284-233-2/11@2011 Ieee.

[13] H.Kusetogullari, "K- Shortest Path Network Problem Solution With A Hybrid Genetic Algorithm: Particle Swarm Optimization Algorithm”, Icton 2011 978-1-4577-0882-4/11@2011 Ieee.

[14] Mingcong Deng, "Optimal Path Planning For Material And Products Transfer In Steel Works Using Aco", Proceedings Of The 2011 international Conference On Advanced Mechatronic Systems 978-0-9555293-7-5/11.

[15] G. Singh, S. Das, S. Pujar, And S. Gosavi, "Ant Colony Algorithms For Steiner Trees: An Application To Routing In Sensor Networks," In Recent Developments In Biologically Inspired Computing, F. J. V. Z. L. N. De Castro, Ed.: Idea Group Publishing, 2004, Pp. 181-206.

[16] G. Chen, T.-D. Guo, W.-G. Yang, And T. Zhao, "An Improved Ant-Based Routing Protocol In Wireless Sensor Networks," In Collaborative Computing: International Conference On Networking, Applications And Worksharing, 2006. Collaboratecom 2006., New York, Ny, 2006, Pp. 1-7.

[17] Y. Zhang, L. D. Kuhn, And M. P. J. Fromherz, "Improvements On Ant Routing For Sensor Networks," M. Dorigo Et Al. (Eds.): Ants 2004, Springer-Verlag Berlin Heidelberg 2004, Vol. Lncs 3172, Pp. 154-165, 2004.

[18] Y. Lu, G. Zhao, And F. Su, "Adaptive Ant-Based Dynamic Routing Algorithm," In In Proceedings Of The 5th World Congress On Intelligent Control And Automation, Hangzhuo, China, 2004, Pp. 2694-2697.

[19] R. G. Aghaei, M. A. Rahman, W. Gueaieb, And A. E. Saddik, "Ant Colony-Based Reinforcement Learning Algorithm For Routing In Wireless Sensor Networks," In Instrumentation And Measurement Technology Conference - Imtc Warsaw, Poland: Ieee, 2007.

[20] Y.-F. Wen, Y.-Q. Chen, And M. Pan, "Adaptive Ant Based Routing In Wireless Sensor Networks Using Energy*Delay Metrics," Journal Of Zhejiang University Science Avol. 9, Pp. 531-538, 2008.

[21] S. Okdem And D. Karaboga, "Routing In Wireless Sensor Networks Using Ant Colony Optimization," In Proceedings Of The First Nasa/Esa Conference On Adaptive Hardware And Systems (Ahs'06), Istanbul, 2006. 